\title{
From inflammation to calcification in atherosclerosis
}

\author{
Takehiro Nakahara $^{1} \cdot$ H. William Strauss ${ }^{2}$
}

Published online: 6 January 2017

(C) Springer-Verlag Berlin Heidelberg 2017

Selecting appropriate therapy for a specific patient with cardiovascular disease requires markers of both disease severity and location to identify the risk of a clinical event. Classic markers, such as the Framingham Risk Score, identify a population at risk, but do not perform well for an individual patient. To get patient specific information at a reasonable cost will likely require a two-step process. Step one would identify the presence of disease with an inexpensive laboratory assay; step two would be a non-invasive imaging technique to localize atheroma at risk of forming an occlusive thrombus. At this time we do not have a sensitive and specific screening blood test. There are, however, multiple imaging techniques that may provide information on the geographic distribution of lesions at moderate or high risk of rupture. PET/CT imaging can identify three markers that may be helpful for the imaging evaluation:

1. Vascular calcification (based on Agatston score of vessel calcification derived from CT);

2. Vascular inflammation (based on focal fluorodeoxyglucose vascular uptake); and

3. Vascular microcalcification (based on focal uptake of ${ }^{18} \mathrm{~F}^{-}$sodium fluoride [1]).

This Editorial Commentary refers to the article doi:10.1007/s00259-0163552-9.

H. William Strauss

harry.strauss@gmail.com

1 Cardiovascular Medicine, Icahn School of Medicine at Mount Sinai, New York, NY, USA

2 Molecular Imaging and Therapy Section, Memorial Sloan-Kettering Cancer Center, New York, NY, USA
Microcalcification $\left({ }^{18} \mathrm{~F}\right.$-fluoride localization in regions with either no CT detectable calcification or small foci of spotty calcification), appears to represent a specific phase in the evolution of an atheroma, when severe inflammation and necrosis begin to stabilize, and microcalcifications form in the necrotic core. This point in the life of the lesion may be a more specific indicator to identify risk for cardiovascular events than imaging inflammation, macrophage cell death [2], or dense calcification [3].

In a recently published manuscript Blomberg et al. [4], challenge the role of ${ }^{18} \mathrm{~F}$-FDG uptake as a cardiovascular disease risk marker, compared to ${ }^{18} \mathrm{~F}-\mathrm{NaF}$ and calcium burden. Their challenge is based on data derived from the CAMONA Study (a multi-institutional study to assess 'Cardiovascular Molecular Calcification Assessed by ${ }^{18} \mathrm{~F}-\mathrm{NaF}$ PET/CT'). The authors performed a retrospective analysis evaluating several findings in the thoracic aorta of these patients in relation to their Framingham Risk Score (FRS). The parameters included: focal uptake of ${ }^{18} \mathrm{~F}-\mathrm{FDG}$, focal uptake of $\mathrm{Na}^{18} \mathrm{~F}$ - fluoride and focal calcification on CT. CAMONA enrolled 89 healthy volunteers (as the control group) and 50 patients with chest pain syndromes. ${ }^{18} \mathrm{~F}$-FDG PET/CT images were recorded $180 \mathrm{~min}$ after intravenous injection of $4.0 \mathrm{MBq} / \mathrm{kg}{ }^{18} \mathrm{~F}$-FDG. ${ }^{18} \mathrm{~F}-\mathrm{NaF}$ PET/CT imaging was performed at $90 \mathrm{~min}$ after intravenous injection of $2.2 \mathrm{MBq} / \mathrm{kg}$ of ${ }^{18} \mathrm{~F}-\mathrm{NaF}$. The interval between FDG and Fluoride imaging was less than 2 weeks. Uptake of ${ }^{18} \mathrm{~F}-\mathrm{FDG},{ }^{18} \mathrm{~F}-\mathrm{NaF}$ and the presence of focal calcifications in the thoracic aorta were quantified by manually delineating regions of interest (ROI). The investigators calculated the maximum decay and background-corrected ${ }^{18} \mathrm{~F}-\mathrm{FDG}$ and ${ }^{18} \mathrm{~F}-\mathrm{NaF}$ activity concentration $(\mathrm{kBq} / \mathrm{ml})$ per slice. Maximum values obtained per ROI were summed and divided by the number of ROIs resulting in a single average maximum value (FDGmax or NaFmax). Calcification was measured in the thoracic aorta on low-dose CT images recorded for 
measuring attenuation at the time of $\mathrm{NaF}$ PET/CT (using an attenuation threshold of 130 Hounsfield units). The calcium volume from all slices was summed and divided by the number of slices resulting in a single mean CT calcium volume $\left(\mathrm{mm}^{3}\right)$.

The investigators report three major observations from this study:

1. Thoracic aorta ${ }^{18}$ F-FDG uptake did not correlate with thoracic aorta ${ }^{18} \mathrm{~F}-\mathrm{NaF}$ uptake or with thoracic aorta $\mathrm{CT}$ calcium burden. On the other hand, thoracic aorta ${ }^{18} \mathrm{~F}-\mathrm{NaF}$ correlated with thoracic aorta CT calcium burden;

2. FRS increased linearly with quartiles of thoracic aorta ${ }^{18} \mathrm{~F}-\mathrm{NaF}$ uptake or thoracic aorta CT calcium burden, although FRS remained similar across quartiles of thoracic aorta ${ }^{18}$ F-FDG uptake;

3. Multivariate linear regression established that thoracic aorta ${ }^{18} \mathrm{~F}-\mathrm{NaF}$ uptake and thoracic aorta $\mathrm{CT}$ calcium burden, but not thoracic aorta ${ }^{18}$ F-FDG uptake, were independent determinants of FRS. From these findings, the investigators concluded that thoracic aorta calcification, but not inflammation, is associated with increased cardiovascular disease risk.
The lack of correlation of vascular FDG uptake and FRS is surprising, and differs from the results of other investigators. Moon et al. [5] found a correlation of carotid FDG uptake and FRS, but not of carotid intima/media ratio, as a predictor of events during an average follow-up of 4.2 years. Similarly, Hetterich et al. [6] found a correlation of FDG vascular uptake with traditional risk factors. The discordance of findings by Blomberg et al. in the CAMONA study population and those of Moon and Hetterich primarily in cancer patients may be due to the difference in the study populations, or other factors. Atherosclerosis is a dynamic, ongoing process. Lesions evolve. Rudd et al. suggest the FDG uptake is stable during a 2 week interval [7]. Lesions evolve, however, from a low risk for causing a clinical event such as a fatty streak, to high risk when the lesion develops a large necrotic core, neovascularization and a thin cap. A high risk lesion may evolve to low risk, with development of a thick cap and a decrease in size of the necrotic core, usually associated with increased calcification within the lesion. Obtaining a 'snapshot' of the status of a dynamic lesion in a small population of patients can lead to erroneous conclusions. The evolution of an atheroma from low risk to high risk and back to low risk is summarized in Fig. 1.

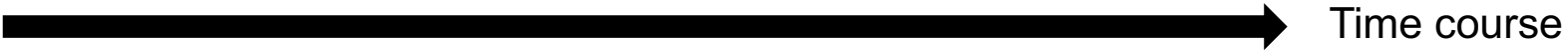

\section{Inflammation}

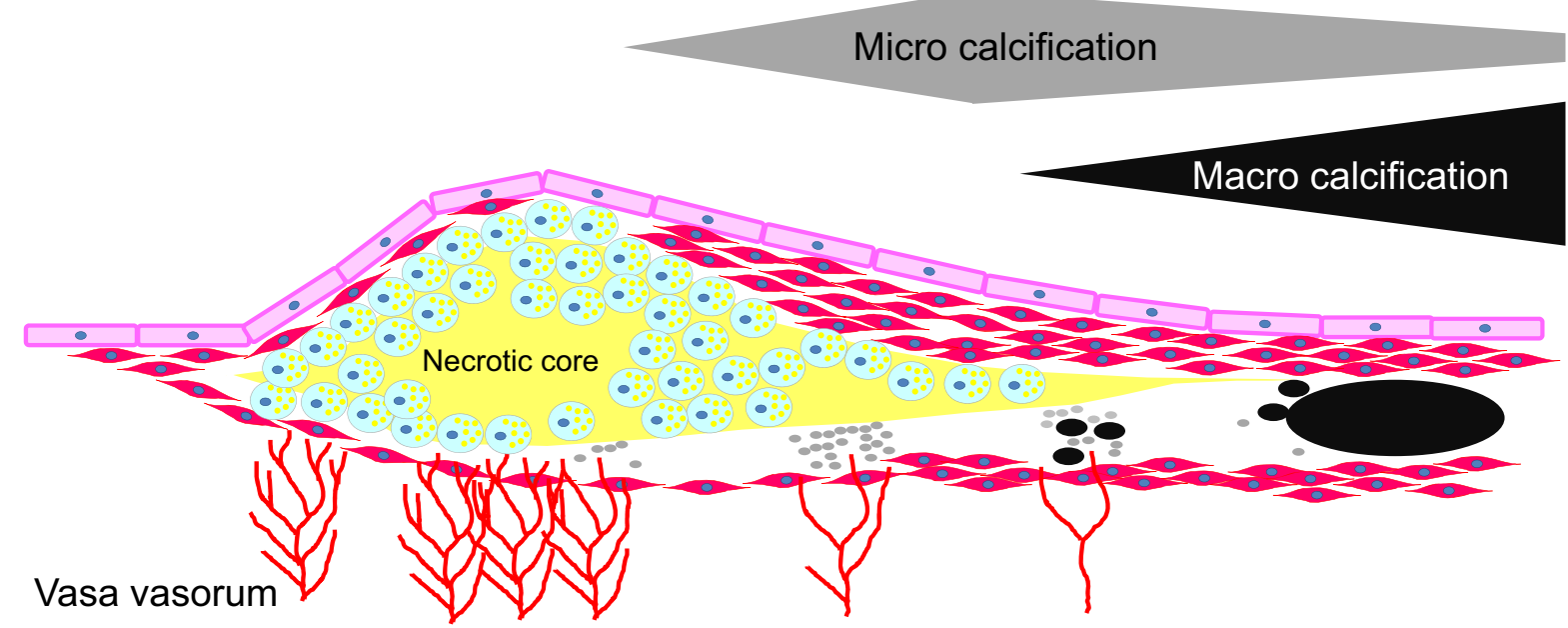

\section{Foam cell : main target of ${ }^{18} \mathrm{~F}-\mathrm{FDG}$ \\ - Macro calcification : main target of CT - Endothelial cell}

Fig. 1 Time course of "inflammation" and "calcification" in atherosclerosis. (overview). Macrophage ingests lipoproteins and become cholesterol-laden foam cells (main target of ${ }^{18} \mathrm{~F}-\mathrm{FDG}$ ). Initially, apoptotic foam cells are phagocytized by adjacent smooth muscle cells. However, as the number of apoptotic cells increase, apoptotic cell debris

\section{Micro calcification : main target of ${ }^{18} \mathrm{~F}-\mathrm{NaF}$ \\ Smooth muscle cell}

is not cleared, resulting in the necrotic core. Vesicles in the necrotic core serve as nucleation sites for calcium phosphate microcalcification (main target of ${ }^{18} \mathrm{~F}$ - NaF). Microcalcifications gather into a large mass or a calcification cascade like bone formation and result in macrocalcification (main target of CT) 
The potential role of ${ }^{18} \mathrm{~F}$-FDG to detect vascular inflammation in atherosclerosis was proposed over 20 years ago [8] and has been well investigated compared to the relatively recent ( 6 year [1]) literature on vascular ${ }^{18} \mathrm{~F}-\mathrm{NaF}$ uptake. ${ }^{18} \mathrm{~F}-\mathrm{FDG}$ imaging has been applied to demonstrate a decrease in carotid inflammation due to therapy with simvastatin [9]. Confounding these changes in FDG uptake is the waxing and waning course of inflammation in atheroma.

In spite of the short-term reproducibility of vascular FDG uptake, both Menezes LJ et al. [10]. and Meirelles et al. [11] confirmed that ${ }^{18}$ F-FDG uptake changes over time, either increasing or decreasing in intensity and extent, reflecting the waxing and waning course of atheroma. On the other hand, calcification had only minimal changes over time, often increasing slightly over the interval between imaging. These changes are in keeping with the intravascular ultrasound findings described by Kubo et al. [12] and eloquently discussed by Arbab-Zadeh and Fuster in their landmark editorial in JACC [13]. The Kubo study determined that of 20 lesions classified as thin cap fibroatheroma on virtual histology intravascular ultrasound (VH-IVUS), 15 healed and five remained unchanged 1 year later.

Although ${ }^{18} \mathrm{~F}-\mathrm{NaF}$ was introduced by Blau et al. in 1962 [14] for detection of osseous metastasis, it was only recently proposed for the detection of vascular microcalcification by Derlin et al. [1]. Derlin et al. showed ${ }^{18} \mathrm{~F}-\mathrm{NaF}$ uptake is a surrogate marker of calcifying carotid plaque and only $6.5 \%$ of arterial lesions seen on the ${ }^{18} \mathrm{~F}$-fluoride images co-localized with ${ }^{18} \mathrm{~F}$ FDG, suggesting that ${ }^{18} \mathrm{~F}-\mathrm{NaF}$ and ${ }^{18} \mathrm{~F}-\mathrm{FDG}$ provide different information about atheroma $[15,16]$. Aortic calcium is a known risk marker of CVD. Fiz F et al. investigated the relationship between the aortic calcium and ${ }^{18} \mathrm{~F}-\mathrm{NaF}$ uptake on atherosclerotic plaque on PET $\mathrm{CT}$ imaging and found greater ${ }^{18} \mathrm{~F}-\mathrm{NaF}$ uptake in the lowest density calcifications $(<210 \mathrm{HU})$ than in medium calcifications (211-510 HU) and dense calcification $(>510 \mathrm{HU})$. They suggested ${ }^{18} \mathrm{~F}-\mathrm{NaF}$ uptake reflects calcium deposition in the early stages of plaque formation [17].

The surprising lack of correlation of FDG uptake and Framingham Risk Score in the Blomberg study needs to be confirmed in a follow-up study with more patients, evaluated at multiple time points, over several years. When we have that data, we will know whether FDG imaging, fluoride imaging or calcium score (or a combination of measurements) provide the most sensitive and specific prognostic information about cardiovascular events.

\section{References}

1. Derlin T, Richter U, Bannas P, et al. Feasibility of $18 \mathrm{~F}$-sodium fluoride PET/CT for imaging of atherosclerotic plaque. J Nucl Med: Off Publ SocNucl Med. 2010;51:862-5.

2. Kolodgie FD, Narula J, Burke AP, et al. Localization of apoptotic macrophages at the site of plaque rupture in sudden coronary death. Am J Pathol. 2000;157:1259-68.

3. van Velzen JE, de Graaf FR, de Graaf MA, et al. Comprehensive assessment of spotty calcifications on computed tomography angiography: comparison to plaque characteristics on intravascular ultrasound with radiofrequency backscatter analysis. J Nucl Cardiol: Off Publ Am Soc Nucl Cardiol. 2011;18:893-903.

4. Björn A, Blomberg PAdJ, Thomassen A, Lam MGE, Vach W, Olsen $\mathrm{MH}$, et al. Thoracic aorta calcification but not inflammation is associated with increased cardiovascular disease risk: results from the CAMONA study. Eur J Nucl Med Mol Imaging. 2016.

5. Moon SH, Cho YS, Noh TS, Choi JY, Kim BT, Carotid LKH, et al. Uptake improves prediction of future cardiovascular events in asymptomatic individuals. J Am Coll Cardiol Img. 2015;8:949-56.

6. Hetterich H, Rominger A, Walter L, et al. Natural history of atherosclerotic disease progression as assessed by (18)F-FDG PET/CT. Int J Cardiovasc Imaging. 2016;32:49-59.

7. Rudd JH, Myers KS, Bansilal S, et al. Atherosclerosis inflammation imaging with 18F-FDG PET: carotid, iliac, and femoral uptake reproducibility, quantification methods, and recommendations. J Nucl Med: Off Publ SocNucl Med. 2008;49:871-8.

8. Vallabhajosula S, Fuster V. Atherosclerosis: imaging techniques and the evolving role of nuclear medicine. J Nucl Med: Off Publ SocNucl Med. 1997;38:1788-96.

9. Tahara N, Kai H, Ishibashi M, et al. Simvastatin attenuates plaque inflammation: evaluation by fluorodeoxyglucose positron emission tomography. J Am Coll Cardiol. 2006;48:1825-31.

10. Menezes LJ, Kayani I, Ben-Haim S, Hutton B, Ell PJ, Groves AM. What is the natural history of $18 \mathrm{~F}-\mathrm{FDG}$ uptake in arterial atheroma on PET/CT? Implications for imaging the vulnerable plaque. Atherosclerosis. 2010;211:136-40.

11. Meirelles GS, Gonen M, Strauss HW. 18F-FDG uptake and calcifications in the thoracic aorta on positron emission tomography/ computed tomography examinations: frequency and stability on serial scans. J Thorac Imaging. 2011;26:54-62.

12. Kubo T, Maehara A, Mintz GS, et al. The dynamic nature of coronary artery lesion morphology assessed by serial virtual histology intravascular ultrasound tissue characterization. J Am Coll Cardiol. 2010;55:1590-7.

13. Arbab-Zadeh A, Fuster V. The myth of the "vulnerable plaque": transitioning from a focus on individual lesions to atherosclerotic disease burden for coronary artery disease risk assessment. J Am Coll Cardiol. 2015;65:846-55.

14. Blau M, Nagler W, Bender MA. Fluorine-18: a new isotope for bone scanning. J Nucl Med: Off Publ SocNucl Med. 1962;3:332-4.

15. Derlin T, Wisotzki $C$, Richter $U$, et al. In vivo imaging of mineral deposition in carotid plaque using $18 \mathrm{~F}$-sodium fluoride PET/CT: correlation with atherogenic risk factors. J Nucl Med: Off Publ SocNucl Med. 2011;52:362-8.

16. Derlin T, Toth Z, Papp L, et al. Correlation of inflammation assessed by 18F-FDG PET, active mineral deposition assessed by 18F-fluoride PET, and vascular calcification in atherosclerotic plaque: a dual-tracer PET/CT study. J Nucl Med: Off Publ SocNucl Med. 2011;52:1020-7.

17. Fiz F, Morbelli S, Piccardo A, et al. (1)(8)F-NaF uptake by atherosclerotic plaque on PET/CT imaging: inverse correlation between calcification density and mineral metabolic activity. J Nucl Med: Off Publ SocNucl Med. 2015;56:1019-23. 